\title{
촉매로서의 $\mathrm{VCl}_{3}$ 를 시용한 $\beta-$ 아세트아마이도 카르보닐 화합물의 간단하고 효율적인 One-Pot 합성

\author{
V. Siddaiah*, G. L. V. Damu, D. Sudhakar, C. Venkata Rao, and V. Christopher

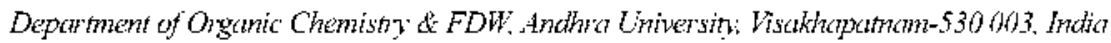
(2008. 9. 22 접수)

\section{$\mathrm{VCl}_{3}$ Catalyzed, A Simple and Efficient One-Pot, Multi-Component Synthesis of $\beta$-Acetamido Carbonyl Compounds}

\author{
V. Siddaiah*, G. L. V. Damu, D. Sudhakar, C. Venkata Rao, and V. Christopher

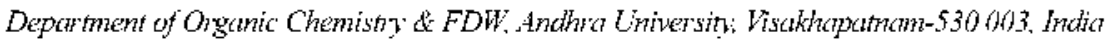

(Received September 22, 2008)

주제어: 방향족 알데히드, 에놀형성 케톤, 염화에세털, 바나듑클로라이드(III), $\beta$-아세트아마이도 카르 보닐 화합물

Keywords: Aromatic Aldehydes, Enolizable Ketones, Acetylchloride, Vanadium(III) Chloride, $\beta$-Acetamido Carbonyl Compounds

\section{INTRODLCTION}

Multi-component reactions (MCRs) are one of the most important protocals in organic synthesis and medicinal chemistry. ${ }^{\prime} \beta$-Acetamido carbonyl compounds are useful building blocks for a number of biologically and pharmaceutically valuable compounds." These are precursors of 1,3-annino alcohols $^{3}$ present antibiotic rikkomycins or neopolyoximes. ${ }^{4}$ Generally these compounds were synthesized through Dakin West reaction. $\beta$-Acetanido ketones have also been synthesized using $\mathrm{Zn}($ II), $\mathrm{Bi}(\mathrm{II}), \mathrm{Sn}(\mathrm{II}), \mathrm{Sc}(\mathrm{III})$ triflates, ${ }^{6} \mathrm{CoCl}_{2},{ }^{7}$ montmorillonite k-10 clay, ${ }^{8} \mathrm{H}_{2} \mathrm{SO}_{4} / \mathrm{SiO}_{2},{ }^{4} \mathrm{BiOCl},{ }^{\text {liv }}$ or heteropoly acid $^{1]}$ as a catalyst. Although these methods are valuable, they suffer from disadvantages such as high temperature, long reaction times, low yields and tedious workup.

In continuation of our work ${ }^{12}$ on the development of useful synthetic methodologies we have investigated a simple and efficient method using a readily available, cheap and non-toxic reagent.
$\mathrm{VCl}_{3}$ is a relatively cheap and non-toxic reagent. It is able to activate carbonyl functionalities for nucleophilic attack and has been used as a Lewis acid for several transformations. ${ }^{13}$ Herein, we report a simple and efficient protocol for the synthesis of $\beta$-acetamido ketones or esters by nulti-component reactions of an aronuatic aldehydes, acetonitrile, an enolizable ketones or $\beta$-ketoesters and acetylchloride in the presence of $10 \mathrm{~mol} \%$ of vanadium (III) chloride (Scheme 1).

\section{RESL:LTS AND DISCLSSION}

To establish the optinual conditions we have carried out the reaction with $p$-methoxybenzaldehyde (1 nunol), acetophenone (l mnol), acetyl chloride (2 nimol) and acetonitrile $(5 \mathrm{~mL})$ under various conditions ( $T a b l e 1$ ). In the absence of catalyst the yield of the product $\beta$-acetamido ketone was $5 \%$ only after $24 \mathrm{l}$. When we used $5 \mathrm{~mol} \%$ of catalyst the yield of the product was found to increase to $75 \%$ witlin $10 \mathrm{~h}$. Further it was observed that when 


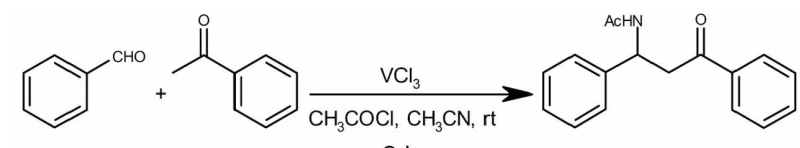

$$
2 \mathrm{~h}
$$

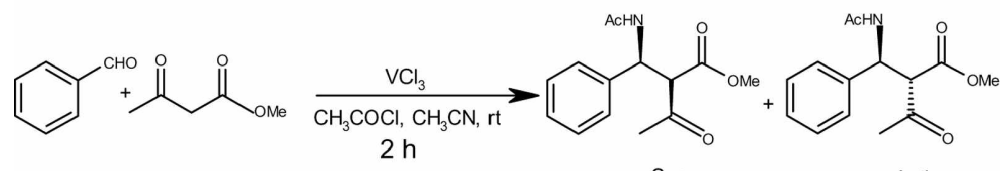

Syn Anti

Solkowe 1.

Table 1. Optimization of the $\mathrm{VCl}_{\text {; }}$ catalyzed multi-component reaction.

\begin{tabular}{|c|c|c|c|}
\hline t.mily & Calalyst $\vee C^{\prime} I_{3}(m)$ & lime (h) & Yicld $(1 / 0)$ \\
\hline 1 & 0 & 24 & 5 \\
\hline 2 & 5 & 10 & 75 \\
\hline 3 & 10 & 2 & 96 \\
\hline
\end{tabular}

Crude yields. the calalysi concentration was increased to $10 \mathrm{~mol} \%$ the product yield increased $1096 \%$ within $2 \mathrm{~h}$.

After optimization various aromatic aldehydes or acetophenones having electron withdrowing as well as electron donating substituents were used for the reaction (Table 2). The conversion was completed within $2 \mathrm{~h}$ at room temperature and the products were obtained in excellent yiclds. 1, 3-diketones formed the corresponding $\beta$-acetamido ketoesters in good yiclds with high diastereoselectivitics. In

Table 2. Synthesis of [3-acelamido carbonyl compounds using samudium chloride

Linly;

"Ratio of the syin and anti isomers (by H NMR)

2008 fid 52 to 6 
Table 2. (Continued) Synthesis of B-acelanido carbonyl componuds using vanadium clulorids

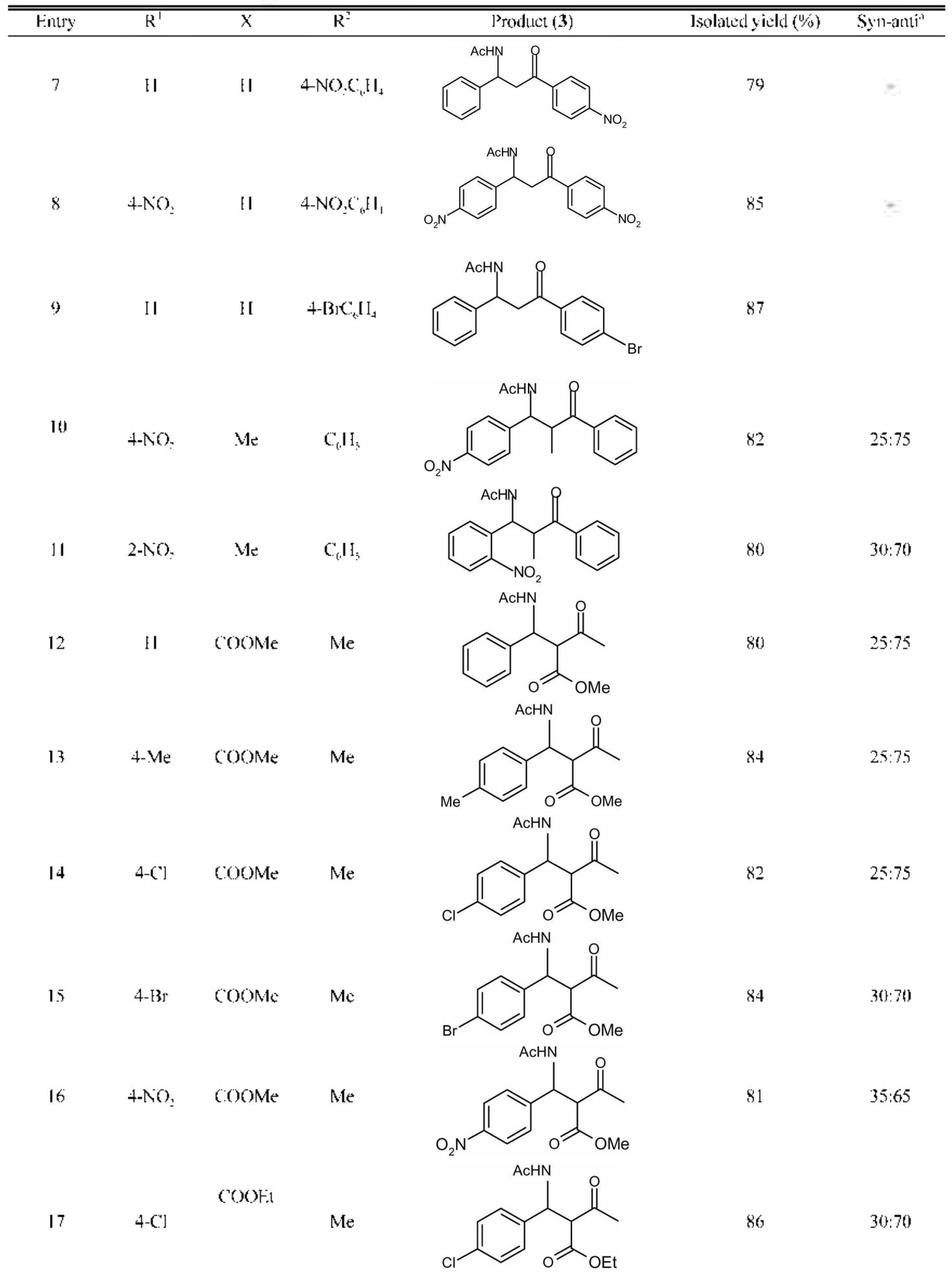

"Ratio of the syn and anti isomers (by '] I .NMR) 
Tuhle 3. Compurison of the results [or the preparation of (3-acetamido ketone (entry: 1, Table 2) using multi-component revetions with ollier catalysts

\begin{tabular}{|c|c|c|c|c|}
\hline$\overline{C \text { Citiolysi }}$ & mol $\%$ & Reaction lime & Reaction lemperature ("C) & Yields $(\%)$ \\
\hline Monmorillonile K-10 & 80 & $7 \mathrm{~h}$ & 70 & 80 \\
\hline $\mathrm{SiO}_{2} \mathrm{HI}_{2} \mathrm{SO}_{4}$ & 78 & $65 \mathrm{~m}$ & 80 & 9) \\
\hline Sc(oli): & 10) & 30) h & $\mathrm{R} 1$ & 82 \\
\hline $\mathrm{CoCl}_{2}$ & 20) & $5 \mathrm{~h}$ & R1 & 90 \\
\hline $\mathrm{BiOCl}$ & 20) & $7 \mathrm{~h}$ & $\mathrm{Rl}$ & 92 \\
\hline VCl; & 10 & $2 \mathrm{~h}$ & $\mathrm{Rl}$ & 96 \\
\hline
\end{tabular}

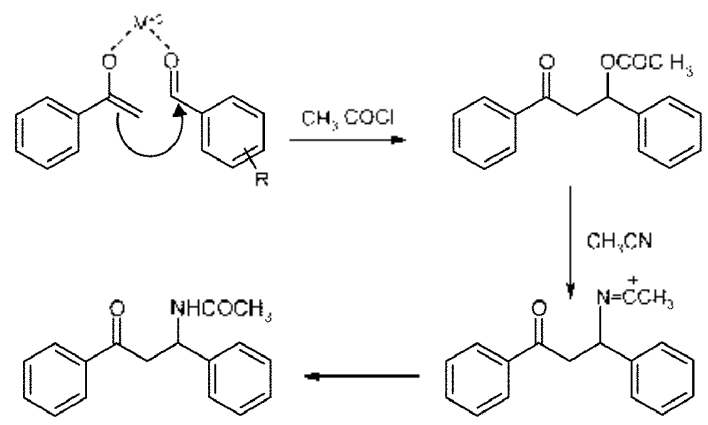

Scheme 2. $\mathrm{A}$ plausible mechanism for the $\mathrm{VCl}_{3}$ calalyod multi-component reaction for the presentation of $\beta$ acelamido ciubonyl compounds.

case ol ketocsters both syn and anti products were bormed (conlimed by ' [ J-NMR). In most of the cases anti isomer was the major produet. All the products were identilied by comparison of analytical data (IR, NMR and MS) of those reported for authentic samples.

As a model reaction, the present method for the preparation of $\beta$-acetamido- $\beta$-(phenyl)-propiophenone (entry 1, Table 2) showed an exeellent efficiency compared to some recently reported procedures (Table 3). Moreover, in the absence of catalyst the reaction proceeds with only little amounts even after $24 \mathrm{~h}$. But the products were obtained in excellent yields within 2 to when the vanadium (III) clloride was used as a catalyst. So we believe that the vanadium chloride activates the aldchyde group for nucleophillic attack and facilitate cnolization (Scheme 2).

\section{CONCLUSIONS}

We have developed an efficient and simple method for the preparation $\beta$-acetamido ketones or esters with $10 \mathrm{~mol}^{2}$ of $\mathrm{VCl}_{3}$. The major advantages of this method include short reaction times, mild reaclion conditions, and casy work up procedure.

\section{EXPERIMENTAL}

Melting points of the compounds were recorded on an electro-thermal apparatus and were uncorrected. Flemental analysis was carried out on CIINS OEA 1108 elemental enalyzer. 'II NMR spectra were recorded on BRUKER AMX-200 spectrometer operating $A T 200 \mathrm{MlIz}$. LC-MS spectra were rocorded on a AGILENT-1100 periods LC.MSD (VL). Starting materials and solvents were puschased Irom Merck or Aldrich.

General procedure for the synthesis of $\beta$-acetamido ketones or esters: A mixlure of aromatic aldehyde (1 mmol), acelophenone or $\beta$-ketoester (1 mmol), acetyl chloride ( $2 \mathrm{mmol}$ ) and $10 \mathrm{~mol} \%$ of $\mathrm{VCl}_{3}$ in acetonitrle $(5 \mathrm{ml})$ was stirred at room temperature. After completion of the reaction as indjcated by 'ILC., the reaction mixture was extracted with dichloromethane. The combined organic layer was concentrated under vacuum and the product was puritied by silica gel column chromatography cluted by an ethyl acetate and hexane (1:1) mixture to afford pure $\beta$-acetamido ketone or an ester in good yicld.

The spectral and analytieal data of some representative $\beta$-acetamido carbonyl compounds are given below.

$\beta$ - $\Lambda$ cetamido- $\beta$-(4-methoxyphenyl)propiophe-

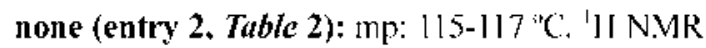
$\left(\mathrm{CDCl}_{3}, 200 \mathrm{Mllz}\right): \delta 1.92$ (s, 3II), 3.20 (dd, $J-$ $7.2,10.0 \mathrm{ll}, 1 \mathrm{H}), 3.63(\mathrm{dd}, J-7.2,10.0 \mathrm{Iz}, 1 \mathrm{H})$, $3.65(\mathrm{~s}, 3 \mathrm{II}), 5.42(\mathrm{~m}, 1 \mathrm{I}), 6.82(\mathrm{~d}, J=8.0 \mathrm{Il} z, 2 \mathrm{I})$, 
$7.25(\mathrm{~d}, J=8.0 \mathrm{~Hz}, 2 \mathrm{H}), 7.49(\mathrm{~s}, 1 \mathrm{H}), 7.52(\mathrm{~m}, 5 \mathrm{H})$; FABMS: $m / 2270[\mathrm{M}+\mathrm{H}]^{-}$; Anal. Calcd. For $\mathrm{C}_{17} \mathrm{H}_{19} \mathrm{NO}_{2}$ : C, 75.80; H, 7.06; N, 5.20. Found: C, 75.76; H, $7.10 ; \mathrm{N}, 4.92$.

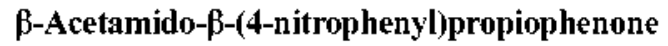
(entry 6, Table 2): mp: $148-130^{\circ} \mathrm{C}$. 'H NMR $\left(\mathrm{CDCl}_{j}, 200 \mathrm{MHz}\right): \delta 2.02(\mathrm{~s}, 3 \mathrm{H}), 3.46(\mathrm{dd}, J=$ $12.0,3.0 \mathrm{~Hz}, 1 \mathrm{H}), 3.79(\mathrm{dd}, J=12.0,2.0 \mathrm{~Hz}, 1 \mathrm{H})$, $5.60(\mathrm{~m}, 1 \mathrm{H}), 6.98(\mathrm{~d}, J=6.0 \mathrm{~Hz}, 1 \mathrm{H}), 7.60-7.42$ $(\mathrm{m}, 5 \mathrm{H}), 7.86(\mathrm{~d}, J=8.0 \mathrm{~Hz}, 2 \mathrm{H}), 8.15(\mathrm{~d}, J=8.0$ $\mathrm{Hz}, 2 \mathrm{H}$ ); FABMS: $m / z 313[\mathrm{M}+\mathrm{H}]^{+}$; Anal. Calcd. For $\mathrm{C}_{1} \mathrm{H}_{16} \mathrm{~N}_{2} \mathrm{O}_{+}$: C, 65.38; H, 5.13; N, 8.97. Found: C, $65.41 ; \mathrm{H}, 5.22 ; \mathrm{N}, 8.92$.

$\beta$-Acetamido- $\beta$-(phenyl)-4-bromopropiophenone (entry 9, Table 2): mp: 97-99 ${ }^{\circ} \mathrm{C}$. 'H NMR (CDCl, $200 \mathrm{MHz}$ ): $\delta 2.16$ (s, $3 \mathrm{H}$ ), $3.4 \mathrm{l}$ (dd, $J=8.20,10.05$ $\mathrm{Hz}, 1 \mathrm{H}$ ), 3.85 (dd, $J=8.2,10.0 \mathrm{~Hz}, 1 \mathrm{H}), 5.40$ (s, $1 \mathrm{H}), 6.81(\mathrm{~d}, J=6.2 \mathrm{~Hz}, 1 \mathrm{H}), 7.40-7.11(\mathrm{~m}, 5 \mathrm{H})$, $7.59(\mathrm{~d}, J=8.2 \mathrm{~Hz}, 2 \mathrm{H}), 7.8 \mathrm{l}(\mathrm{d}, J=8.2 \mathrm{~Hz}, 2 \mathrm{H})$; FABMS: $m / z$ 346, $348[\mathrm{M}+\mathrm{H}]^{-}$; Anal. Calcd. For $\mathrm{C}_{1} \mathrm{H}_{16} \mathrm{NO}_{2} \mathrm{Br}: \mathrm{C}, 58.95 ; \mathrm{H}, 4.62 ; \mathrm{N}, 4.05$. Found: C, $58.90 ; \mathrm{H}, 4.65 ; \mathrm{N}, 4.09$.

Methyl-2-acetyl-3-acetamido-3-(p-methyl)propionate (entry 13, Table 2) (anti): mp: $112-114^{\circ} \mathrm{C}$. ${ }^{\text {'}} \mathrm{H}$ NMR ( $\left.\mathrm{CDCl}_{3}, 200 \mathrm{MHz}\right): \delta 2.05$ (s, 3H), 2.18 $(\mathrm{s}, 3 \mathrm{H}), 2.32(\mathrm{~s}, 3 \mathrm{H}), 3.70(\mathrm{~s}, 3 \mathrm{H}), 4.06(\mathrm{~d}, J=5.90$ $\mathrm{Hz}, 1 \mathrm{H}), 5.72(\mathrm{~m}, 1 \mathrm{H}), 6.90(\mathrm{~d}, J=9.3 \mathrm{~Hz}, \mathrm{lH})$, 7.21-7.05 (m, 4H); FABMS: $n / z 278[\mathrm{M}+\mathrm{H}]^{-}$; Anal. Calcd. For $\mathrm{C}_{15} \mathrm{H}_{19} \mathrm{NO}_{4} ; \mathrm{C}, 64.98 ; \mathrm{H}, 6.86 ; \mathrm{N}, 5.05$. Found: C, 64.9l; H, 6.90; N, 5.12.

Acknowledgements. The authors thank the Head, NMR divison IICT, Hyderabad, for providing Spectral data.

\section{REFERENCES}

1. (a) Weber, L. Ding Discovery Totay 2002, 7, 143, (b) Domling, A. Cum Opin. Chem. Biol. 2002, 6, 306. (c)
Weber, L.; Illegen, K.; Almestetter, M. Swnletr 1999. 366. (d) Armstrong, R. W.; Combs, A. P.; Tempest, P. A.; Brown, S. D.; Keating, T. A. Acc. Chem. Res. 1996, 29, 123. (e) Lgi, I.; Werner, B.; Domling, A. Molectles 2003, 8, 53. f Donilling, A. Chm: Opin. Chem. Biol. 2000, 4, 318 .

2. Casimir, J. R; Turetta, C.; Ettouati, L.; Paris, J. Tetrahedran Lett. 1995, 36, 4797.

3. (a) Enders, D.; Moser, M.; Geibel, G.; Laufer, M. C. Symthesis 2004, 2040. (b) Barluenga, J.; Viado, A. L.; Agnilar, E.; Fustero, S.; Olano, B. J. Org. Chem. 1993, 58,5972

4. (a) Kobinata, K.; Lramoto, M.; Nishii, M.; Kusakabe, H.; Naksmura, G; Isono, K. Agri. Biol. Chem. 1980, 44, 1709. (b) Daeln, LI.; Hagenmaier, H.; Hoelne, H.; Koenig, W. A; Wolf, G; Zaelner, H. Arch Mcrobiol. 1976, 107, 249.

5. Dakin, H. D.; West, R. J. Biol. Chem. 1938, 78, 745.

6. Pandey, G; Singh, R. P.; Garg, A.; Singh, V. K. Tetrahedron Lett. 2005, 46, 2137.

7. Rao, I. N.; Prabhakaran, E. N.; Das, S. K.; Iqbal, T. $J$. Org. Chem. 2003, 68, 4079 .

8. Bahulayan, D.; Das, S. K.; Iqbal, I. J. Org. Chem. 2003, 68, 5735 .

9. Khodaei, M. M.; Khostopour, A. R.; Fattah pour, P. Tetrahedron Lett. 2005, 46, 2105.

10. Ghosh, R.; Maiti, S.; Chakraborty, A. Symlert 2005, 1 , 115.

11. Rafiee, E.; Tork, F.; Joshaghani, M. Bioorgonic. Med. Chem. Letr. 2006, 16, 1221 .

12. (a) Maheswara, M.; Siddaiah, V; Damu, G L. V; Venkata Rao, C. Joumal of Molecular Catalysis A: Chemical. 2006, 255, 49. (b) Malheswara, M.; Siddaiah, V; Koteswara Rao, Y; Yew-Min Tzeng Y. M.; Sridlar, C. Journal of Molecular Catalusis A: Chemical. 2006, 260, 179. (c) Das, B.; Venkateswarlu, K.; Majliv, A; Siddaiah, V; Ravinder Reddy, K. Joumal of Holecular Catalusis 4: Chemical 2007, 267, 30.

13. Suril Kumar, B.; Kumar, P. S.; Srinivasulu, N.; Rajita, B.; Thirupathi Reddy, Y; Narasimha Reddy, P.; Ldupi, R. H. Chemistry of Heterocyclic Compounds 2006, 42 , 171. 